\title{
A Data Acquisition System for Tele-Health Monitoring
}

\author{
Ashwini A. Mankari ${ }^{1}$, D. S. Bhosale ${ }^{2}$ \\ ${ }^{1}$ Savitirbai Phule Pune University, JSPM’s BSIOTR E\&TC, Pune Nagar Highway, Wagholli, India \\ ${ }^{2}$ Professor, JSPM's Bhivarabai Sawant Institute of Technology and Research, Savitirbai Phule Pune University, \\ Pune Nagar Highway, Wagholli, India
}

\begin{abstract}
In this paper, the physiological parameters such as ECG, Pulse rate and Temperature are obtained, processed using ARM7 LPC 2138 processor and displayed in a MATLAB graphical user interface. If any vital parameter goes out of normal range then alert SMS will be sent to Doctor Mobile. And to transmit ECG data to physicians for monitoring, diagnosis and patients care at a significantly low cost, regardless of patient's location. Now a days Telehealth improves clinical outcomes and reduces acute care hospitalizations because its process and core technologies enable careful, 24-hour monitoring of patients by healthcare professionals from a remote point.
\end{abstract}

Keywords: ECG, Pulse Rate, ARM, MATLAB.

\section{Introduction}

The electronics technology has entered almost in all aspects of day-to-day life, and the medical field is not exception for that. The need for well-equipped hospitals and diagnostic centers is increasing day by day as the people are becoming more conscious about their health problems. The medical world today faces two basic problems when it comes to patient monitoring, frrstly the need of healthcare providers present bedside the patient and secondly the patient is restricted to bed and wired to large machines. In order to achieve better quality patient care, the above cited problems have to be solved.

As the technologies are advancing it has become feasible to design to horne based vital sign monitoring system to display, record and transmit signals from human body to any other location. The computer based Signal Acquisition, processing and analysis system using MATLAB to display ECG Waveform and filtering tool for ECG waveform. In this paper discusses the aspects of acquisition of physiological Parameters like ECG, Temperature, Pulse rate, preprocessing them and displaying them in a graphical user interface for being viewed by the doctor and also observe the clinically useful data. This system is expected to monitor patient under critical care more conveniently and accurately for diagnosing which can be interfaced with GPRS to bring it under a internetwork for the doctor to monitor the patient's condition sitting in his own Hospital without being physically present near to the patient's bed.

\section{Proposed Work}

The proposed system contains Sensors for measurement of various body parameters such as ECG, PPG, and Temperature. The system contains the ARM7 LPC2148 processor for data processing and the processed data is sent over the GPRS and then displayed to the GUI using MATLAB10. If any parameter goes abnormal then the system will sent an alert SMS to the doctor through GSM

modem. The physician viewed by the data in two ways. Firstly on Personal Computer using Remote Desktop sharing and secondly on Android mobile having application of Remote desktop sharing. This system reduces costs by enabling in-horne monitoring of patients, eliminating the need for utilization of expensive facilities, and reducing the need for transportation of patients to physicians and medical centers.

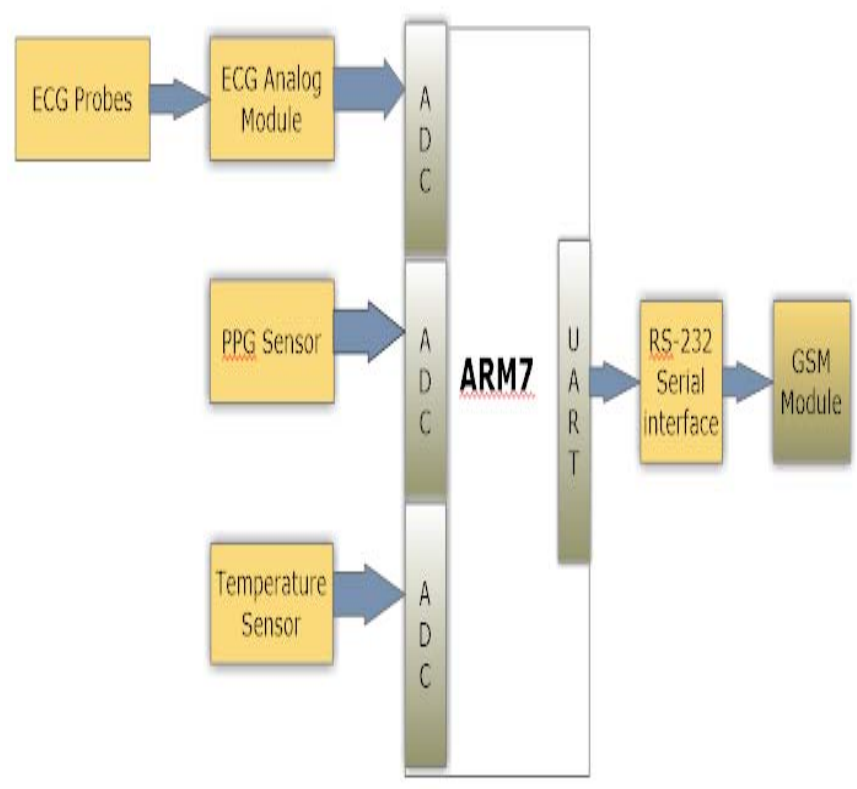

Figure 1: Block diagram for Tele-health monitoring system

\section{Design Methodology}

ECG signal is plotted as per the flowchart shown in fig. The results are carried out on subject with sleeping position along with three electrodes, one for each parameter ECG, Temperature and PPG. The GPRS modem sim 900 is used to upload the parameter file to the GPRS. The doctor from the remote end uses this parameter data and processes data in MATLAB.

\section{Volume 5 Issue 2, February 2016}




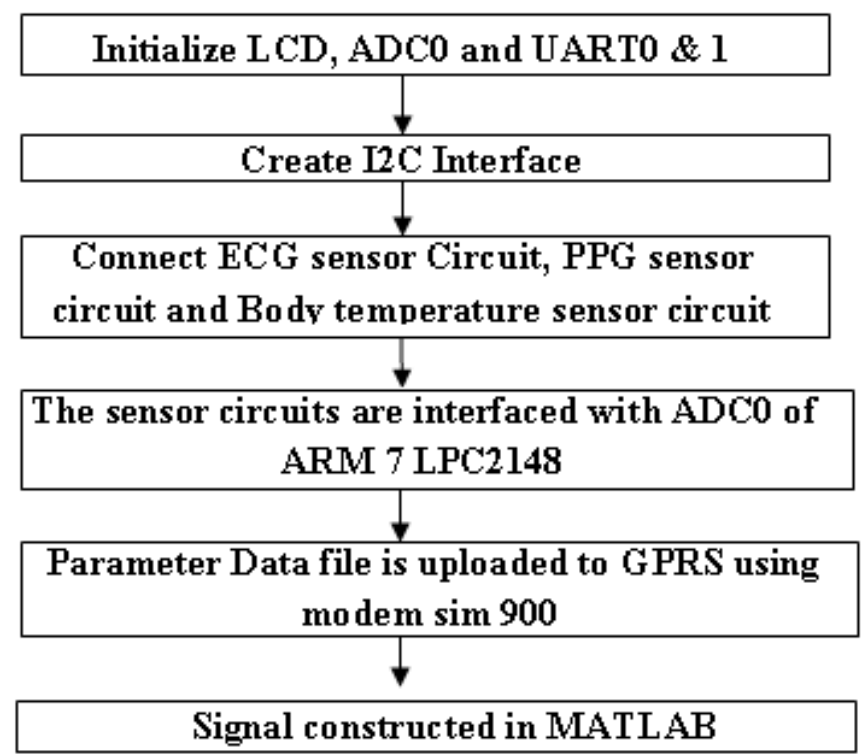

Figure 1 Flow chart of Data execution

The ECG signal is extracted from the ECG sensor and amplified as shown in figure 3 . This amplified signal is then digitized using ADC. Digitized ECG data is then transmitted using GPRS wireless technology. On the parallel lines at the receiving end the ECG, Temperature and PPG data is received using GPRS technology and displayed on PC of doctor for analysis.

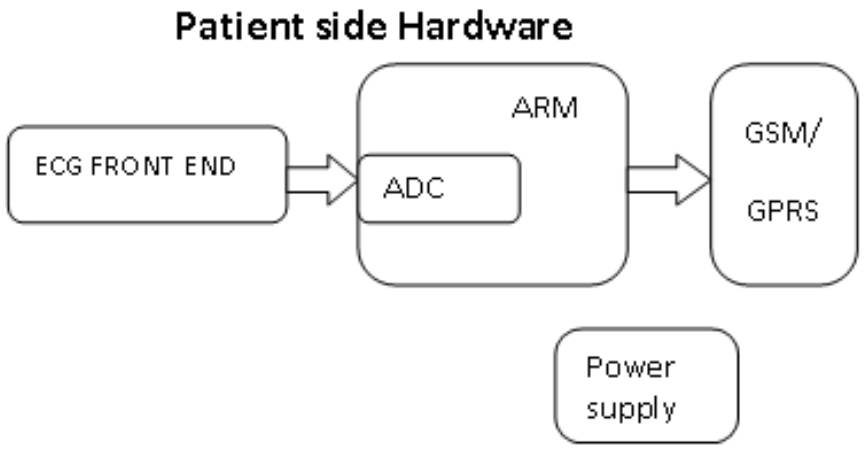

Figure 3: Patient side module for transmission of ECG signal

Function and specification of proposed system is to provide a real time 3-lead ECG trace on a P.C. A simple intuitive GUI that may be used by a person with no medical background. Transmission of data from remote unit is initiated from sensor node. Easy setup and installation of entire system. Two separate units. A portable unit that is attached to the person being monitored. A second unit interfaces directly to the P.C and acquires data that is being transmitted wirelessly from first unit. Received data can be visualized on PC.

\section{Result and Discussion}

The real time ECG signal is obtained to analyze the authenticity and precision of the system. The results are carried out on subject with sleeping position along with three electrodes. The signal output from signal detection hardware system is processed in MATLAB.

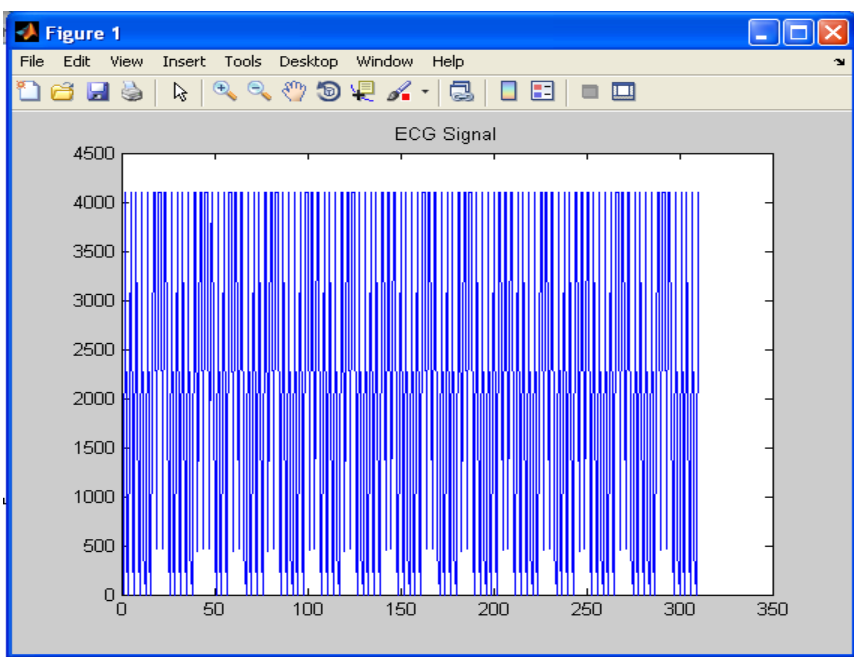

Figure 4: ECG signal at electrode

\section{Conclusion}

The proposed system is used to develop a Data Acquisition system to measure ECG, PPG and Body Temperature of the patient. It involve microcontroller for obtaining and processing the signal. Thus hardware implementation of system allows to fabricate the portable device. Interfacing of BI signal with ARM opens door for new development.ARM handles both the controlling of analogue measurements and storing the measurement samples. MATLAB is used to processing data.

A novel noninvasive technique for ECG, PPG and Body Temperature of the patient measurement is successfully implemented. The system is tested for resistance and results obtained are satisfactory. Since, human body has impedance. It can be detected using high end electrodes.

\section{References}

[1] Mohamed Fezari, Mounir Bousbia-Salah, and Mouldi Bedda "Microcontroller Based Heart Rate Monitor" The International Arab Journal oflnfonnation Technology, Vol. 5, No. 4, October 2008

[2] J. Yoo, L. Yan, S. Lee, Y. Kim, and H. J. Yoo, "A 5.2 $\mathrm{mW}$ self-configured wearable body sensor network controller and a $12 \mathrm{uW}$ wirelessly powered sensor for a continuous health monitoring system," IEEE J. SolidState Circuits, vol. 45, no. 1, pp. 178-188, Jan. 2010.

[3] Liang Kai, Xu Zhang, Yuan Wang, Huang Suibiao, Guan Ning, "A system of portable ECG monitoring based on Bluetooth mobile phone" IEEE international Symposium on IT in medicine and education" Dec 2011.

[4] Enhancement of real-time multi-patient monitoring system based on wireless sensor networks, International Journal of Physical Sciences Vol. 6(4), pp. 664-670, 18 February, 2011.

\section{Author Profile}

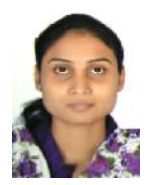

Ashwini Mankari received the B.E degree in Electronics and Telecommunication Engineering from Visveshwarayya Institute of Technology Belgum Karnataka, in 2011.She is now pursuing Masters of 
Engineering from Savitribai Phule Pune University since 2013 under the guidance of Prof. D. S. Bhosale. Her specialization area is VLSI and Embedded System.

Prof. Dilip. S. Bhosale working as an Associate Professor in JSPM's BSIOTR, Pune since 2011.He has total experience of 22 years in the field of E\&TC. He has received his Masters of Engineering from Mumbai University. His specialization lies in VLSI \& Embedded System and also in Image processing. 